Artículo

\title{
Tendencias en la precipitación diaria de la cuenca alta Laja-Peñuelitas, Guanajuato
}

\author{
Jorge Jaimes Rodríguez ${ }^{1 \S}$ \\ Laura Alicia Ibáñez Castillo ${ }^{1}$ \\ Gustavo Antonio Arévalo Galarza ${ }^{2}$ \\ Mario Alberto Vázquez Peña ${ }^{1}$ \\ Alejandro Ismael Monterroso Rivas ${ }^{2}$
}

${ }^{1}$ Posgrado en Ingeniería Agrícola y Uso Integral del Agua-Universidad Autónoma Chapingo. Carretera México-Texcoco km 36.5, Chapingo, Estado de México, México. CP. 56230. (jorgejrdz@gmail.com; libacas@gmail.com). ${ }^{2}$ Departamento de Suelos-Universidad Autónoma Chapingo. Carretera México-Texcoco km 36.5, Chapingo, Estado de México, México. CP. 56230.(arevalogg@gmail.com; mvazquezp@correo.chapingo.mx).

§Autor para correspondencia: jorgejrdz@gmail.com

\section{Resumen}

Detectar cambios en el comportamiento de la precipitación, temperatura o cualquier variable atmosférica es fundamental para los tomadores de decisiones. El identificar tendencias en el comportamiento histórico de las estaciones meteorológicas brinda información para el comportamiento futuro del clima. El presente trabajo analiza los cambios en los extremos de precipitación en la cuenca alta Laja-Peñuelitas en el estado de Guanajuato, utilizando los índices de cambio climático desarrollados por el equipo de expertos en detección e índices de cambio climático. Se analizó un periodo de 36 años (1982-2017) utilizando datos diarios de precipitación, seleccionando estaciones meteorológicas convencionales con menos de $25 \%$ de datos perdidos, sometiendo estas bases de datos a un proceso de control de calidad de datos y homogeneización, obteniendo 11 índices de precipitación a través del programa RClimDex. Los resultados muestran una tendencia estadística no significativa $(62.09 \%)$ y significativa ascendente $(14.05 \%)$, indicando que en la cuenca alta Laja-Peñuelitas, es posible establecer el mantenimiento y ligero aumento en el comportamiento y cantidad de la precipitación.

Palabras clave: cambio climático, homogeneización RClimDex.

Recibido: abril de 2021

Aceptado: agosto de 2021 


\section{Introducción}

La implementación exitosa de las estrategias de adaptación al cambio climático depende en gran medida de la detección precisa y oportuna de los cambios del clima a nivel regional y local en diferentes partes del mundo (Adger et al., 2005). En algunas regiones de México, los efectos esperados del cambio climático serán un incremento de temperatura mayor que $3{ }^{\circ} \mathrm{C}$ y una disminución en la precipitación del orden de 15\%, estos cambios del clima, en esas regiones, disminuirán el escurrimiento superficial y la recarga de acuíferos lo que hará peor los problemas del crecimiento poblacional y económico (Martínez y Patiño, 2012). En algunas otras regiones del país es posible que se presenten tendencias del clima diferentes, debido a las características del área y factores antropogénicos. Para detectar, por regiones y en cuencas específicas, esos cambios climáticos, es necesario calcular índices de cambio climático para regiones específicas de México.

Los índices derivados de los datos diarios son un intento de extraer información objetivamente de las observaciones meteorológicas que responden a preguntas sobre los extremos que afectan a muchos sistemas humanos y naturales (Zhang et al., 2011). El equipo de expertos en detección e índices de cambio climático (IPCC) ha coordinado un conjunto de 11 índices de precipitación y 16 índices de temperatura, adoptados desde el cuarto informe de evaluación (AR4) del IPCC (Yan et al., 2014). Para calcular los índices se requieren series de tiempo de variables climáticas largas y sin discontinuidades (Persson et al., 2007). Estos pueden ser calculados en el programa RClimDex (Zhang y Yang, 2004b).

En este trabajo se tiene interés en determinar la tendencia de la precipitación en la cuenca alta LajaPeñuelitas; a través, de la generación de índices de cambio climático en precipitación. Dicho interés surge ante la necesidad de estar preparados en el espacio y tiempo de la cuenca, ya sea para enfrentar inundaciones o adaptarse a las sequías. Las inundaciones y las sequías pueden afectar la producción agrícola y la seguridad de la población. En este trabajo, la zona de estudio tiene una disponibilidad de 36 años de datos diarios de temperatura y lluvia.

\section{Materiales y métodos}

Área de estudio. La cuenca alta Laja-Peñuelitas para este estudio fue definida hasta la estación hidrométrica 12715 Puente Dolores (Figura 1). Está delimitada dentro de las coordenadas $101^{\circ}$ $31^{\prime} 60.00^{\prime \prime}$ latitud oeste y $100^{\circ} 51^{\prime}$ '60.00" longitud oeste; $21^{\circ} 6^{\prime} 0.00^{\prime}$ ' latitud norte y $21^{\circ} 33^{\prime} 60.00^{\prime}$ " longitud oeste, cubriendo una superficie de $1683 \mathrm{~km}^{2}$, de acuerdo con INEGI (2010) se encuentra ubicada en el estado de Guanajuato, México, en la región hidrológica número 12 Lerma-Santiago y en específico en la parte alta de la subcuenca RH12Ha Laja - Peñuelitas. La altitud en la zona de estudio varía de los 2963 a los $1890 \mathrm{msnm}$. El río de La Laja cruza la parte oriental de la ciudad de Dolores Hidalgo, Guanajuato.

La mayor parte de la cuenca $(61 \%)$ cuenta con un clima semiseco templado, siendo el restante templado subhúmedo (INEGI, 2008). La precipitación anual promedio es de $558 \mathrm{~mm}$, siendo concentrada mayormente en los meses de junio a octubre. Los usos del suelo y vegetación predominantes en la cuenca alta Laja-Peñuelitas son la vegetación de pastizal natural-inducido (34\%) y la agricultura de temporal (30\%) (INEGI, 2016). Los suelos Feozem, en su mayoría arcillosos, representan $70 \%$ del área de la cuenca de estudio (INEGI, 1998). 


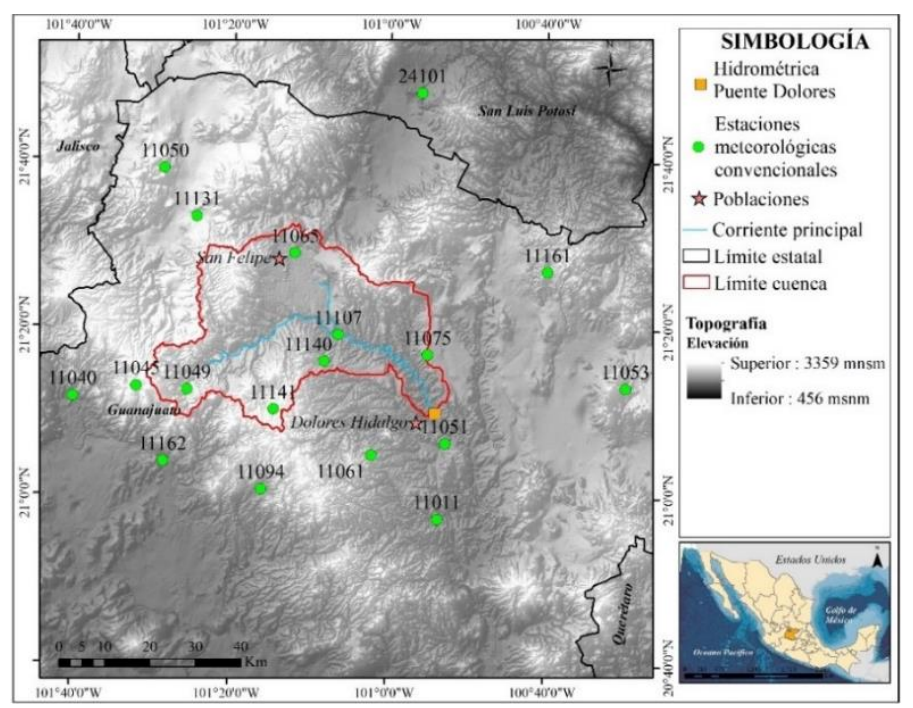

Figura 1. Ubicación de la cuenca alta Laja-Peñuelitas.

\section{Datos climáticos y fuentes}

Se utilizó información climática del Servicio Meteorológico Nacional a través de estaciones climatológicas convencionales dentro y cercanas a la cuenca (SMN, 2019). Se realizó un primer filtro seleccionando las estaciones que no rebasaron más de $25 \%(<25 \%)$ de datos perdidos de precipitación (Arriaga y Cavazos, 2010) esto efectuado para un periodo de 36 años continuos (1982-2017). Un segundo filtro consistió en la verificación de la continuidad de la serie de datos, eliminando las estaciones climatológicas que poseían un año o más sin información como lo aplicado por Zarazúa et al. (2014), omitiendo este criterio para aquellas estaciones que por su ubicación eliminarlas representaba un vacío de información para una área específica de la cuenca.

Un tercer filtro fue la eliminación de estaciones meteorológicas redundantes, esto generado por la proximidad de estas en una misma área geográfica. El control de calidad de los datos es un paso necesario antes del análisis de la variación de temperatura y precipitación porque los valores atípicos erróneos pueden afectar seriamente las tendencias (Yan et al., 2014). El control de calidad fue realizado usando el programa RClimDex (Zhang y Yang, 2004b), para cada base de datos de las estaciones meteorológicas seleccionadas. El control de calidad consistió en identificar valores atípicos como cantidades de precipitación diaria menores que cero, valores de precipitación demasiado grandes, problemas de redondeo, etc.

La homogeneización de los datos consistió en 1) verificación de los cambios partiendo de la presencia de estos en cada una de las estaciones objetivo vs comportamiento de estos las estaciones meteorológicas cercanas; 2) el análisis de los valores de la prueba estadística PFMAx; y 3) la justificación de los cambios debido a fenómenos climáticos externos tales como el Niño/Niña. Una vez detectados los puntos de cambio se realizó el proceso de homogeneización de la serie de datos con ajuste por cuantiles QM (Quantile Matching Algorithm) en los casos donde las series no resultaron homogéneas (Wang y Feng, 2013). La información sobre cómo el software detecta inconsistencias y efectúa el proceso de homogeneización es proporcionada por Wang y Feng (2013). Las estaciones analizadas se presentan en el (Cuadro 1). 
Cuadro 1. Estaciones meteorológicas consideradas para la cuenca alta Laja-Peñuelitas.

\begin{tabular}{|c|c|c|c|c|c|c|c|}
\hline Núm. & Clave & Nombre & $\begin{array}{l}\text { Latitud } \\
\text { (grados) }\end{array}$ & $\begin{array}{c}\text { Longitud } \\
\text { (grados) }\end{array}$ & $\begin{array}{l}\text { Altitud } \\
\quad(\mathrm{m})\end{array}$ & $\begin{array}{c}\text { Datos perdidos } \\
1982-2017(\%)\end{array}$ & Homogeneizada \\
\hline 1 & 11011 & Cinco Señores & 20.958 & -100.893 & 2062 & 2.06 & $\mathrm{Si}$ \\
\hline 2 & 11040 & Los Castillos & 21.195 & -101.668 & 1865 & 0.27 & \\
\hline 3 & 11045 & Media Luna & 21.217 & -101.534 & 2221 & 4.22 & \\
\hline 4 & $11049^{*}$ & $\begin{array}{c}\text { Nuevo Valle } \\
\text { Moreno }\end{array}$ & 21.211 & -101.426 & 2247 & 2.09 & $\mathrm{Si}$ \\
\hline 5 & 11050 & Ocampo & 21.65 & -101.480 & 2253 & 3.00 & \\
\hline 6 & 11051 & Peñuelitas & 21.108 & -100.878 & 1906 & 0.53 & \\
\hline 7 & 11053 & Pozos & 21.220 & -100.496 & 2206 & 9.99 & $\mathrm{Si}$ \\
\hline 8 & 11061 & San Antonio & 21.084 & -101.034 & 2090 & 12.51 & \\
\hline 9 & $11065^{*}$ & $\begin{array}{l}\text { San Felipe } \\
\text { (DGE) }\end{array}$ & 21.484 & -101.2 & 2100 & 24.75 & \\
\hline 10 & 11075 & Soledad nueva & 21.283 & -100.917 & 2011 & 21.61 & $\mathrm{Si}$ \\
\hline 11 & 11094 & $\begin{array}{l}\text { Guanajuato } \\
\text { (DGE) }\end{array}$ & 21.014 & -101.266 & 2008 & 3.26 & \\
\hline 12 & $11107^{*}$ & La Quemada & 21.323 & -101.107 & 2003 & 13.71 & \\
\hline 13 & 11131 & Las Trojes & 21.555 & -101.409 & 2198 & 13.46 & \\
\hline 14 & $11140^{*}$ & El Carbón & 21.269 & -101.136 & 2115 & 3.82 & $\mathrm{Si}$ \\
\hline 15 & $11141^{*}$ & $\begin{array}{l}\text { Ciénega de } \\
\text { Negros }\end{array}$ & 21.173 & -101.242 & 2475 & 1.34 & \\
\hline 16 & 11161 & El Vergel & 21.45 & -100.664 & 2192 & 3.95 & $\mathrm{Si}$ \\
\hline 17 & 11162 & Comanjilla & 21.068 & -101.475 & 1898 & 1.38 & \\
\hline 18 & 24101 & Villa de Reyes & 21.804 & -100.933 & 1820 & 12.55 & $\mathrm{Si}$ \\
\hline
\end{tabular}

*= estaciones dentro de la cuenca; ${ }^{* *}=$ para la variable precipitación.

\section{Tendencias en precipitación}

Se utilizó el software RClimDex (Zhang y Yang, 2004b), para estimar las tendencias en precipitación (Cuadro 2). El procedimiento seguido fue el indicado en Zhang y Yang (2004b) empezando por hacer ajustes en el número de días que se rebasa un valor de lluvia 'nn', Rnn. Los valores de lluvia a ser superados, 'nn' y analizados, en acorde a los valores que suelen presentarse en la zona, fueron los siguientes: 1) $22.3 \mathrm{~mm}$; 2) $37.3 \mathrm{~mm}$; 3) $30 \mathrm{~mm}$;) $40 \mathrm{~mm}$; 5) $50 \mathrm{~mm}$; 6 ) 60 $\mathrm{mm}$; y 7) $70 \mathrm{~mm}$. Lo último ya que el rango de $70 \mathrm{~mm}$ a $150 \mathrm{~mm}$ de lluvia máxima acumulada en 24 horas es catalogado como lluvia intensa y también usado por el Centro Nacional de Prevención y Desastres (CENAPRED) en México en su clasificación de los diferentes tipos de precipitación (Prieto et al., 2010). 
Cuadro 2. Definiciones de los índices de cambio climático en precipitación*.

\begin{tabular}{|c|c|c|c|}
\hline Índice & Descripción del nombre & Definición & Unidades \\
\hline RX1day & $\begin{array}{l}\text { Cantidad máxima de } \\
\text { precipitación en un día }\end{array}$ & Máximo anual de precipitación en 1 día & $\mathrm{mm}$ \\
\hline RX5day & $\begin{array}{l}\text { Cantidad máxima de } \\
\text { precipitación en } 5 \text { días }\end{array}$ & $\begin{array}{l}\text { Máximo anual de precipitación en } 5 \text { días } \\
\text { consecutivos }\end{array}$ & $\mathrm{mm}$ \\
\hline SDII & $\begin{array}{l}\text { Índice simple de } \\
\text { intensidad diaria }\end{array}$ & $\begin{array}{l}\text { Precipitación anual total dividida para el } \\
\text { número de días húmedos (definidos por la } \\
\text { precipitación } \geq 1 \mathrm{~mm} \text { ) en un año }\end{array}$ & $\mathrm{mm} \mathrm{d}^{-1}$ \\
\hline $\mathrm{R} 10$ & $\begin{array}{l}\text { Número de días con } \\
\text { precipitación intensa }\end{array}$ & $\begin{array}{l}\text { Número de días en un año en que la } \\
\text { precipitación } \geq 10 \mathrm{~mm}\end{array}$ & $\mathrm{~d}$ \\
\hline $\mathrm{R} 20$ & $\begin{array}{l}\text { Número de días con } \\
\text { precipitación muy } \\
\text { intensa }\end{array}$ & $\begin{array}{l}\text { Número de días en un año en que la } \\
\text { precipitación } \geq 20 \mathrm{~mm}\end{array}$ & $\mathrm{~d}$ \\
\hline Rnn & $\begin{array}{l}\text { Número de días sobre nn } \\
\qquad \mathrm{mm}\end{array}$ & $\begin{array}{l}\text { Número de días en un año en que la } \\
\text { precipitación } \geq \mathrm{nn} \text { mm }\end{array}$ & $\mathrm{d}$ \\
\hline CDD & Días secos consecutivos & $\begin{array}{l}\text { Número máximo de días secos } \\
\text { consecutivos con } \mathrm{RR}<1 \mathrm{~mm}\end{array}$ & d \\
\hline CWD & $\begin{array}{l}\text { Días húmedos } \\
\text { consecutivos }\end{array}$ & $\begin{array}{l}\text { Número máximo de días húmedos } \\
\text { consecutivos con } R R \geq 1 \mathrm{~mm}\end{array}$ & $\mathrm{~d}$ \\
\hline R95p & Días muy húmedos & $\begin{array}{c}\text { Precipitación anual total en que RR> } 95 \\
\text { percentil }\end{array}$ & $\mathrm{mm}$ \\
\hline R99p & $\begin{array}{l}\text { Días extremadamente } \\
\text { húmedos }\end{array}$ & $\begin{array}{c}\text { Precipitación anual total en que RR> } 99 \\
\text { percentil }\end{array}$ & $\mathrm{mm}$ \\
\hline PRCPTOT & $\begin{array}{c}\text { Precipitación total anual } \\
\text { en los días húmedos }\end{array}$ & $\begin{array}{c}\text { Precipitación anual total en los días } \\
\text { húmedos }(\mathrm{RR} \geq 1 \mathrm{~mm})\end{array}$ & $\mathrm{mm}$ \\
\hline
\end{tabular}

\section{Resultados y discusión}

En la Figura 2 se presenta el número de estaciones meteorológicas $(\mathrm{n}=18)$ y las tendencias positivas o negativas observadas en los índices de cambio climático definidos en el Cuadro 2. Los resultados muestran tanto aquellos índices con tendencia significancia estadística $(p \leq 0.05)$, así como de los que se mantienen con una pendiente de la recta $=0$ (sin tendencia) para el periodo 1982-2017. Los resultados de la (Figura 2) fueron clasificados en cinco categorías $(p \leq 0.05)$ de acuerdo con: $(+)$ incremento no significativo, (++) incremento significativo, (-) decremento no significativo, (--) decremento significativo y $(\mathrm{S} / \mathrm{T})$ sin tendencia significativa. En la parte inferior izquierda de la (Figura 2), se muestran los símbolos de dicha tendencia estadística. 


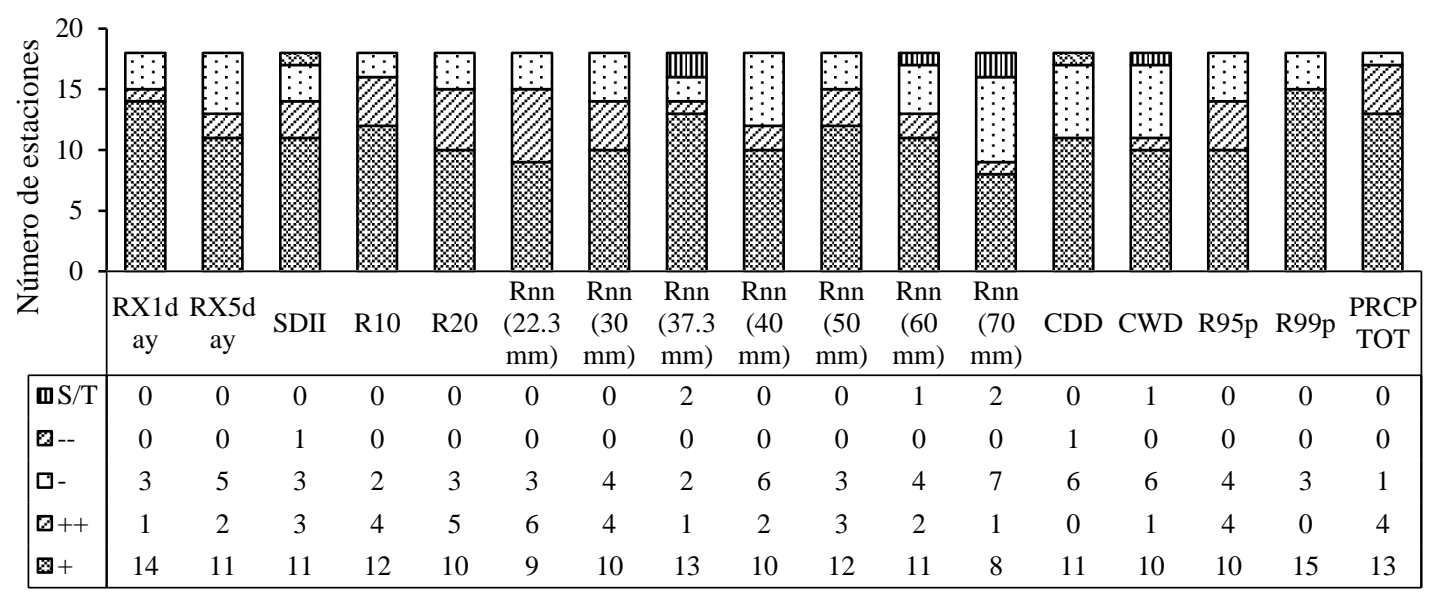

Figura 2. Tendencias en los índices para las 18 estaciones meteorológicas en la cuenca alta LajaPeñuelitas.

Se observó la presencia de tres tipos de tendencias: no significativa ascendente (62.09\%), no significativa descendente $(21.24 \%)$ y significativa ascendente (14.05\%). Este fenómeno mixto encontrado también en Aguilar et al. (2005) al existir un aumento no significativo de la precipitación, con patrones espaciales muy mixtos de tendencias positivas y negativas cuando se estudiaron las estaciones individuales. En la (Figura 3) se muestra la distribución espacial de las estaciones meteorológicas que presentaron tendencia significativa (incremento o decremento) en el valor máximo anual de lluvia en 24 h (RX1day).

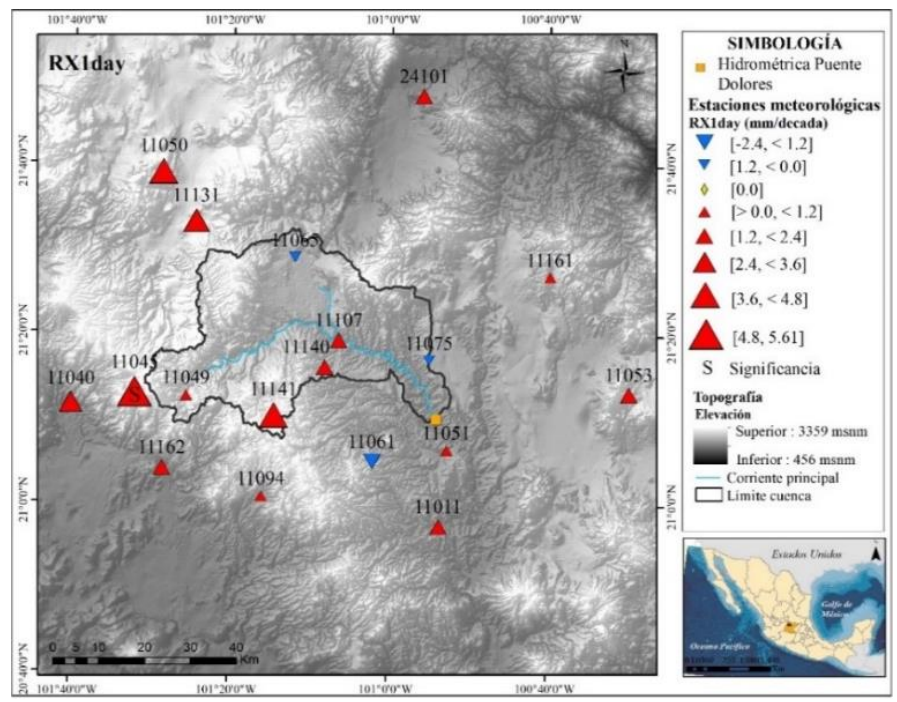

Figura 3. Tendencia del índice de lluvia máxima en 24 h (RX1day).

El índice RX1day solo presentó tendencia ascendente estadísticamente significativa para la estación 11045 Media Luna con $5.61 \mathrm{~mm}^{\text {década }}{ }^{-1}$, ubicada del lado del municipio de León, Guanajuato, en la parte alta de la cuenca (aproximadamente a $2470 \mathrm{msnm}$ ). En el resto de las estaciones meteorológicas de la parte alta (Las Trojes, Salvatierra, y del lado de San Miguel de Allende, Guanajuato) existe una tendencia ascendente no significativa generalizada. 
Al analizar las tendencias de índices climáticos, si el referente son los criterios generales para el país, se pudiera concluir que no hay tendencias significativas ascendentes en la lluvia máxima en $24 \mathrm{~h}$, pero si se analiza desde el punto de vista de las desviaciones estándar de los propios datos de la cuenca bajo estudio, se observará lo siguiente. En la Figura 4 se muestra un análisis para cuatro índices de cambio climático basados en ver su variación con respecto a la desviación estándar, alrededor de cada círculo se observan las claves de las estaciones meteorológicas y los círculos son que tanto se alejan en desviaciones estándar de las medias aritméticas.

Por ejemplo, en la Figura 4a se muestran los valores promedio anuales del índice RX1day y su ubicación respecto a las desviaciones estándar $(\sigma)$ de la media de los valores de las estaciones de la cuenca. Desde el punto de vista geográfico de la cuenca, las estaciones 11 011, 11 040, 11 094, 11141 presentaron un incremento por encima del promedio de dos desviaciones estándar y la estación 11131 con una disminución de dos desviaciones estándar para el índice RX1day. La (Figura 4b), (Figura 4c y Figura 4d) muestran un análisis similar, basado en desviaciones estándar, para los índices de precipitación total anual, número de días con lluvia mayor o igual a $70 \mathrm{~mm}$ y número de días con lluvia mayor o igual a $20 \mathrm{~mm}$.

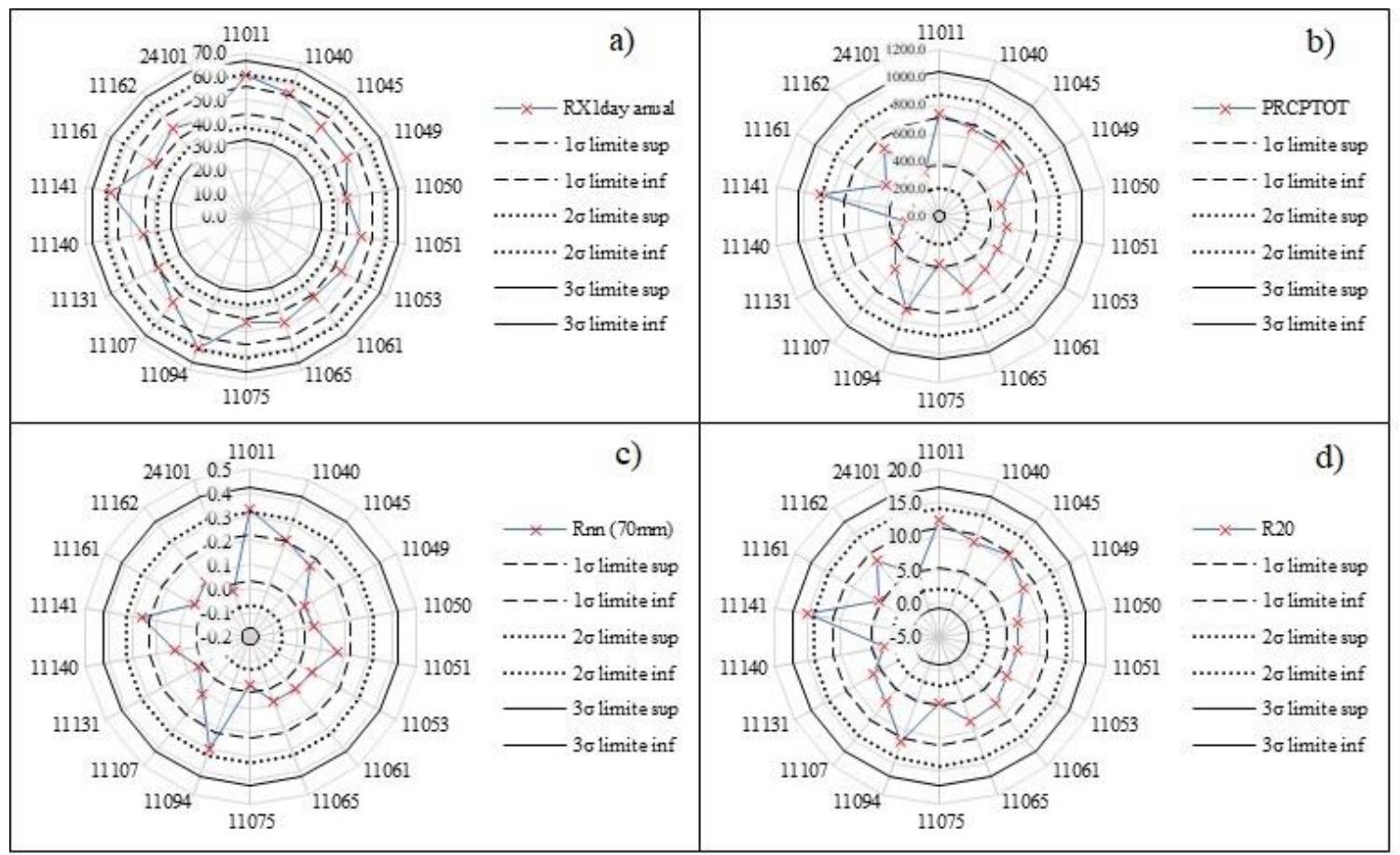

Figura 4. Valores promedio de los índices RX1day (a); PRCPTOT (b); Rnn (70 mm) (c); y R20 (d).

En la Figura 5 se observa la distribución espacial de las estaciones meteorológicas que presentaron tendencia significativa en la cuenca alta Laja-Peñuelitas para los índices de cambio climático RX5day (cantidad máxima de precipitación en 5 días), SDII (intensidad diaria de la lluvia), PRCPTOT (precipitación total anual en los días húmedos) y Rnn (70 mm). 


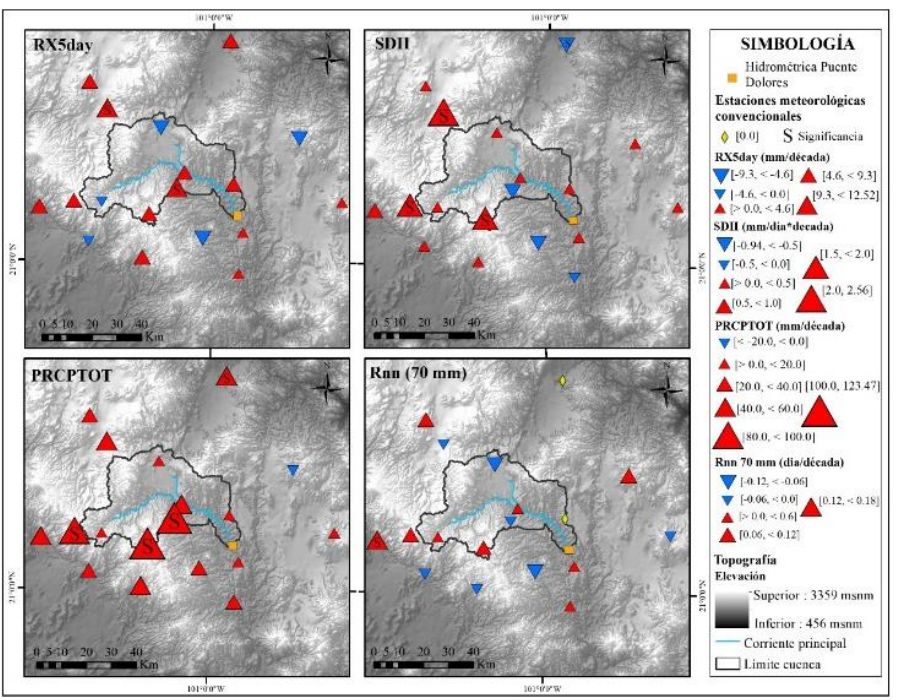

Figura 5. Tendencia de las estaciones meteorológicas ante los índices RX5day (superior izquierda); SDII (superior derecha); PRCPTOT (inferior derecha); Rnn 70 mm (inferior derecha).

Resulta importante analizar la intensidad de la lluvia, porque en general se comenta que, por el cambio climático, los eventos de lluvia serán más intensos, se analiza la lluvia máxima en cinco días, porque es un criterio al momento de calcular escurrimientos en una cuenca. En la Figura 5, en general, hay un mayor incremento en la tendencia de los índices de intensidad y de lluvia anual; un poco de incremento en lluvia en cinco días y en general, no hay cambio significativo de número de días con lluvia igual o mayor a $70 \mathrm{~mm}$, el cual ya es un valor de una gran lluvia en esta cuenca.

Como contraste a lo que ocurre en esta zona de Guanajuato, Aguilar et al. (2005) Encontraron para zonas en el Norte de México, que el PRCPTOT no presentó una tendencia significativa, a pesar de que el número de estaciones con tendencias positivas fue mayor, encontrándose un número considerable de pendientes negativas, agrupadas alrededor de las estaciones más al norte de México $\mathrm{y}$ alrededor de las ubicadas en el lado suroeste del istmo centroamericano promediando estos patrones una tendencia positiva no significativa de $8.7 \mathrm{~mm}_{\text {década }}{ }^{-1}$ de 1961 a 2003.

El índice Rnn $(70 \mathrm{~mm})$ presentó tendencia ascendente estadísticamente significativa para la estación 11040 (0.16 días década ${ }^{-1}$ ), presentándose un fenómeno mixto de reducción e incremento no significativo para la cuenca. En la Figura 6 se ilustra la distribución espacial de las estaciones meteorológicas que presentaron tendencia significativa en la cuenca alta Laja-Peñuelitas para los índices de cambio climático R10, R20, Rnn $(22.3 \mathrm{~mm})$ y Rnn $(37.3 \mathrm{~mm})$. El índice R10 mostró una tendencia ascendente estadísticamente significativa para las estaciones 11045 (4.58 días década $^{-1}$ ), 11140 (3.78 días década ${ }^{-1}$ ), 11141 (4.41 días década ${ }^{-1}$ ) y 24101 (2.08 días década ${ }^{-1}$ ).

Se observa que el índice R20 presentó una influencia regional en la parte media-alta de la cuenca, marcada por la tendencia ascendente estadísticamente significativa para las estaciones 11040 (1.29 días década ${ }^{-1}$ ), 11045 (3.44 días década ${ }^{-1}$ ), 11131 (1.93 días década ${ }^{-1}$ ), 11140 (1.81 días década $^{-1}$ ) y 11141 (2.73 días década ${ }^{-1}$ ), que lo convierte en el segundo índice con mayor cantidad de estaciones significativas; indicando que el número de días en un año con lluvia fuerte basado en el criterio de Prieto et al. (2010) se está incrementando, esto tendrá un importante efecto en el futuro en el aumento del número de escurrimientos con caudales elevados. 


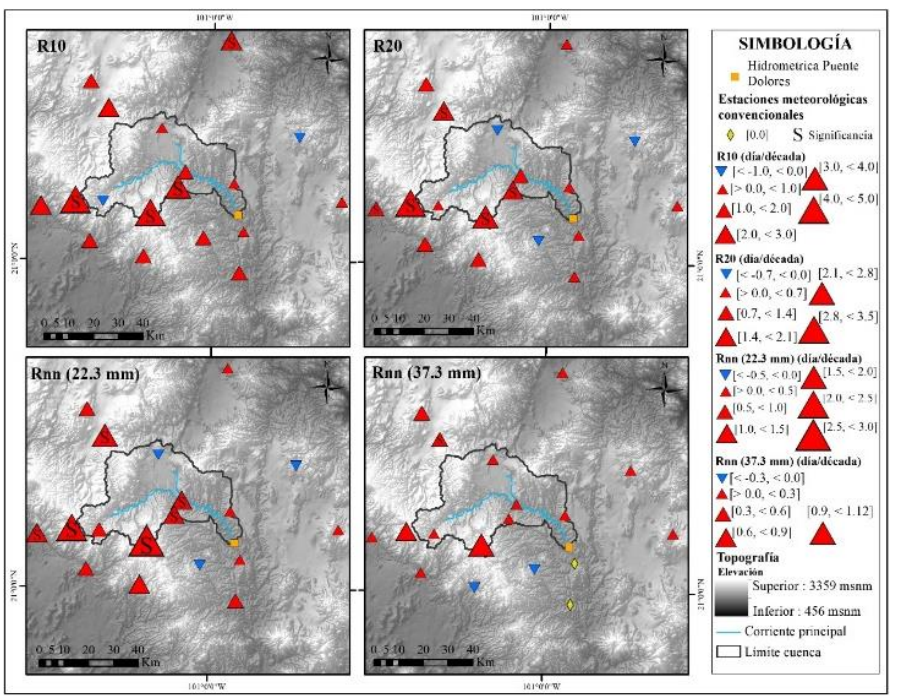

Figura 6. Tendencia de las estaciones meteorológicas ante los índices R10 (superior izquierda); R20 (superior derecha); Rnn 22.3 mm (inferior derecha); Rnn 37.3 mm (inferior derecha).

De los 11 índices de cambio climático en precipitación para la cuenca alta Laja-Peñuelitas, el índice Rnn (22.3 mm) fue el que más estaciones con tendencia ascendente estadísticamente significativa obtuvo, con las estaciones 11040 (1.12 días década ${ }^{-1}$ ), 11045 (2.11 días década ${ }^{-1}$ ), 11107 (1.24 días década $\left.{ }^{-1}\right), 11131$ (1.8 días década $\left.{ }^{-1}\right), 11140$ (1.23 días década $\left.{ }^{-1}\right)$ y 11141 (2.99 días década ${ }^{-}$ ${ }^{1}$ ), marcado por la estación 11107 ubicada al centro de la cuenca, identificándose un patrón regional de incremento en la parte media-alta de la cuenca.

Kachok y Ivanova (2019) calcularon diversos índices para la Reserva de la Biosfera El Vizcaino en Baja California Sur durante el periodo 1960-2012 presentando el índice CWD una tendencia al aumento al igual que R20 y PRCPTOT, este último fenómeno que se repite en la cuenca alta Laja-Peñuelitas. En los estados más cercanos al área de estudio, como lo presentado por Núñez y García (2018) para 48 estaciones del estado de Jalisco próximo a la cuenca alta Laja-Peñuelitas para el periodo 1980-2010 calcularon cinco índices de precipitación, identificando tendencias positivas para los índices: PRCPTOT (54\% de la estaciones) con una tendencia promedio de 7.2 mm década ${ }^{-1}$, índice SDII (77\%) y una tendencia promedio de $0.4 \mathrm{~mm}_{\text {día }}{ }^{-1}$ decada $^{-1}$, RX1day $(73 \%)$ con $3.9 \mathrm{~mm}$ década $^{-1}$, CDD $(85 \%)$ con 11.6 días década ${ }^{-1}$, presentando estos índices para la cuenca alta Laja-Peñuelitas en la tendencia general el mismo comportamiento.

En contraparte con tendencia negativa el índice CWD (67\%) presentando un valor promedio de -0.4 días década ${ }^{-1}$, este fenómeno siendo diferente para la cuenca en el cual se presentó una mayor cantidad de eventos positivos que negativos. Pita y Ortega (2020) para Zacatecas, cercano a la cuenca alta Laja-Peñuelitas, para el periodo (1976-2015), el índice PRCPTOT mostró un incremento con valores significativos en ciertas áreas (una de ellas, la zona más cercana a la cuenca de estudio), esto concordando con los resultados de este trabajo, en el cual a pesar de no ser un fenómeno completamente significativo, la tendencia es al incremento, el análisis de tendencias espaciales indicó que los CDD aumentaron en todo el estado, con la excepción de dos áreas muy locales, una de ellas cercana a la cuenca alta Laja-Peñuelitas, presentando estas áreas pendientes negativas. 
Sin embargo, la significancia estadística espacial y temporal de este índice fue baja, no obstante, en la cuenca de estudio y en especial en la parte norte de la misma el fenómeno tiende al incremento, aunque siendo no significativo; finalmente identificaron un aumento de la precipitación máxima diaria (RX1day) en los últimos años, comportamiento que se presenta de igual manera en la cuenca alta Laja-Peñuelitas aunque de manera no significativa. Ruiz et al. (2020) obtuvieron 11 índices de cambio climático para el estado de Aguascalientes, cercano en dirección este a la cuenca alta Laja-Peñuelitas en un rango de $70 \mathrm{~km}$, encontrando en pequeñas zonas del este y norte del estado un aumento en las precipitaciones en 1 y 5 días (RX1day y RX5day), en el este y en el sur aumentaron tanto la intensidad de las lluvias como el número de días con fuertes precipitaciones; en una zona muy pequeña al este, la precipitación total anual también muestra evidencia de un aumento.

Dichos comportamientos en la región este del estado concordantes con los resultados obtenidos en este estudio. Martínez y Patiño (2012) mencionan que en las latitudes en las que se ubica México, los efectos esperados del cambio climático será una disminución en la precipitación; sin embargo, el presente trabajo muestra un incremento (significativo o no significativo) de la lluvia, esto principalmente visualizado a través de los índices PRCPTOT, R10, R20 y Rnn para la zona de estudio.

Pita y Ortega (2020) señalan que los resultados son generalmente consistentes cuando se analizan lugares o áreas adyacentes con condiciones climáticas similares, pero pueden diferir para cuencas hidrográficas remotas o regiones con diferentes condiciones climáticas, un ejemplo de ello es lo mostrado por Velasco et al. (2015), los cuales empleando dos estaciones meteorológicas cercanas a 38 km en los límites del estado de Puebla y Tlaxcala el periodo de 1970 a 2012 encontraron para el índice PRCPTOT incremento y decremento, a pesar de su corta distancia. Kotlarski et al. (2017); Zittis (2017) de la misma manera indican, que las tendencias relacionadas con las precipitaciones no son claras y existen diferencias significativas de un país a otro, lo que indica que los cambios globales en esta variable climática no son homogéneos en todo el mundo.

\section{Conclusiones}

El presente estudio fue diseñado para identificar los cambios y tendencias en los extremos climáticos en la cuenca alta Laja-Peñuelitas utilizando índices extremos relacionados con la precipitación para el período 1982-2017. Los índices de cambio climático son herramientas viables que permiten la obtención de tendencias a partir de registros históricos y con estas proyectar tendencias futuras de incremento o decremento en precipitación, que se pueden utilizar como insumo para estudios que tengan como objetivo determinar cambios en el clima y los efectos de estos en materia hidrológica, biológica, social, etc.

El análisis climático de la cuenca alta Laja-Peñuelitas mostró tendencias en extremos climáticos de precipitación no estudiados previamente en esta área particular de México, destacando que, la cuenca alta Laja-Peñuelitas presenta la tendencia general al incremento de los fenómenos de lluvia. Es recomendable que futuros estudios en la cuenca alta Laja-Peñuelitas se oriente tanto al comportamiento futuro de la precipitación a través de la utilización de modelos de circulación general (GCM), así como a la obtención de los índices de cambio climático en temperatura y con ello generar un mejor panorama en el comportamiento de los extremos climáticos. 
Los índices de cambio climático (lluvia anual total, lluvia máxima en 24 h, intensidad diaria de la lluvia, lluvia en cinco días consecutivos y número de días con lluvia intensa y muy intensa) mostraron una tendencia de ascenso en la cuenca alta Laja-Peñuelitas, esto último resulta de gran importancia para considerar en el diseño de obras hidráulicas y en materia de prevención de inundaciones.

\section{Agradecimientos}

Al Consejo Nacional de Ciencia y Tecnología (CONACYT) por el apoyo otorgado para la realización de este artículo.

\section{Literatura citada}

Adger, W. N.; Arnell, N. W. and Tompkins, E. L. 2005. Adapting to climate change: perspectives across scales. Global Environmental Change. 15(2):75-76. https://doi.org/10.1016/ j.gloenvcha.2005.03.001.

Arriaga, R. S. and Cavazos, T. 2010. Regional trends of daily precipitation indices in northwest México and southwest united states. J. Geophys. Res. 115(D14):1-10. https://doi.org/10.1029/2009jd013248.

INEGI. 1998. Conjunto de datos vectoriales de la carta edafológica, escala 1:250 000, serie i. Instituto Nacional de Estadística y Geografía. Aguascalientes, México. https://www.inegi.org.mx/app/biblioteca/ficha.html?upc=702825234621.

INEGI. 2008. Conjunto de datos vectoriales, unidades climáticas, escala 1:1 000 000. Instituto Nacional de Estadística y Geografía. Aguascalientes, México. https://www.inegi.org.mx/ app/biblioteca/ficha.html?upc $=702825267568$.

INEGI. 2010. Siatl: simulador de flujos de agua de cuencas hidrográficas 3.2. Instituto Nacional de Estadística y Geografía. http://antares.inegi.org.mx/analisis/red_hidro/siatl/\#

INEGI. 2016. Conjunto de datos vectoriales de uso del suelo y vegetación, escala 1:250 000, serie VI (Capa Unión). Instituto Nacional de Estadística y Geografía. Aguascalientes, México. https://www.inegi.org.mx/app/biblioteca/ficha.html?upc=889463598459.

Kachok, R. e Ivanova, A. 2019. Índices de cambio climático en la reserva de la biosfera El Vizcaíno, Baja California Sur, México (1960-2012). Rev. Brasil. Climatol. 24(15):67-93.

Kotlarski, S.; Szabó, P.; Herrera, S.; Räty, O.; Keuler, K.; Soares, P. M.; Cardoso, R. M.; Bosshard, T.; Pagé, C.; Boberg, F.; Gutiérrez, J. M.; Isotta, F. A.; Jaczewski, A.; Kreienkamp, F.; Liniger, M. A.; Lussana, C. and Pianko, K. 2017. Observational uncertainty and regional climate model evaluation: a pan-European perspective. Inter. J. Climatol. 39(9):3730-3749. https://doi.org/10.1002/joc.5249.

Martínez, A. P. F. y Patiño, G. C. 2012. Efectos del cambio climático en la disponibilidad de agua en México. Tecnología y Ciencias del Agua. 3(1):5-20.

Núñez, G. G. y García, S. J. 2018. Análisis de la calidad de datos y la tendencia de algunos índices de precipitación en el estado de Jalisco. Rev. Internac. Estadís. Geograf. 9(2):14-27.

Persson, G.; Bärring, L.; Kjellström, E.; Strandberg, G. and Rummukainen, M. 2007. Climate indices for vulnerability assessments. Swedish Meteorological and Hydrological Institute. S-601 76 Norrköping, Sweden. Report RMK Num. 111. 64 p. 
Prieto, G. R.; Avendaño, G. M. A. y Matías, R. L. G. 2010. Tormentas severas. In: serie fascículos. Centro Nacional de Prevención de Desastres-Secretaría de Seguridad y Protección Ciudadana. $1^{\text {a }}(\mathrm{Ed})$. Ciudad de México. 54 p.

SMN. 2019. Base de datos climatológica. Servicio Meteorológico Nacional. https://smn.conagua.gob.mx/.

Vázquez, A. J. L. 2010. Guía para el cálculo y uso de índices de cambio climático en México. Instituto Nacional de Ecología- Secretaría de Medio Ambiente y Recursos Naturales. $1^{\mathrm{a}}$ (Ed.). México, DF. 88 p.

Velasco, H. M.; Morales, A. T.; Estrella, C. N. G., Díaz, R. R.; Juárez, S. J. P.; Hernández, V. M. y Bernal, M. R. 2015. Tendencias y variabilidad de índices de cambio climático: enfoque agrícola en dos regiones de México. Rev. Mex. Cienc. Agríc. 6(7):1587-1599.

Wang, X. L. and Feng, Y. 2013. RHtests_dlyPrcp user manual. Climate research division, atmospheric science and technology directorate, science and technology branch, environment Canada. Toronto, Ontario, Canada. 17 p.

Yan, G.; Qi, F.; Wei, L.; Aigang, L.; Yu, W.; Jing, Y.; Aifang, C.; Yamin, W.; Yubo, S. and Qianqian, M. 2014. Changes of daily climate extremes in loess plateau during 1960-2013. Quaternary International. 1-17. https://doi.org/10.1016/j.quaint.2014.08.052.

Zarazúa, V. P.; Ruiz, C. J. A.; Ramírez, O. G.; Medina, G. G.; Rodríguez, M. V. M.; De la Mora, O. C.; Flores, L. H. y Durán, P. N. 2014. Índices de extremos térmicos en las llanuras costeras del Golfo Sur en México. Rev. Mex. Cienc. Agríc. (10):1843-1857.

Zhang, X.; Alexander, L.; Hegerl, G. C.; Jones, P.; Tank, A. K.; Peterson, T. C.; Trewin, B. and Zwiers, F. W. 2011. Indices for monitoring changes in extremes based on daily temperature and precipitation data. Wires climate change. 2(6):851-870. https://doi.org/ 10.1002/wcc. 147.

Zhang, X., and Yang, F. 2004a. RClimDex (1.0) manual del usuario. (Traduc.). Santos, J. L. Centro internacional para la investigación del fenómeno del Niño Ciifen. Guayaquil, Ecuador. 22 p.

Zhang, X. y Yang, F. 2004b. Rclimdex (1.0) user manual. Climate Research Branch, Environment Canada. Downsview, Ontario, Canada. 23 p.

Zittis, G. 2017. Observed rainfall trends and precipitation uncertainty in the vicinity of the Mediterranean, middle East and North Africa. Theor. Appl. Climatol. 134(3-4):1207-1230. https://doi.org/10.1007/s00704-017-2333-0. 